\title{
Fase de vida livre e flutuação sazonal do Boophilus microplus em Botucatu, São Paulo, Brasil
}

\section{The free-living phase and seasonal variation of Boophilus microplus in Botucatu, São Paulo State, Brazil}

\author{
Vamilton Alvares Santarém ${ }^{1 *}$; Izidoro Francisco Sartor ${ }^{2}$
}

\begin{abstract}
Resumo
Em um intervalo de três anos, estudou-se as fases de vida livre e parasitária do Boophilus microplus, no município de Botucatu, São Paulo, Brasil. Mensalmente, fêmeas ingurgitadas foram distribuídas em pastagem de Brachiaria decumbens e mantidas no meio ambiente. Determinou-se correlação negativa dos períodos de pré-postura e postura com a temperatura do ar, umidade relativa e precipitação pluviométrica. A postura foi registrada durante todo o estudo, existindo, porém, alta redução na eclosão de ovos nos meses de maio a agosto. Os períodos mínimos e máximos médios de sobrevivência larval foram observados, respectivamente, nas progênies de teleóginas expostas em agosto e março. A dinâmica populacional do B. microplus foi estudada pela contagem, em intervalos de 14 dias, de fêmeas ingurgitadas maiores que 4,5 mm sobre o lado esquerdo de um grupo de nove bovinos mestiços de raças de origem européia e indiana, de ambos os sexos e com aproximadamente dois anos de idade. Os piques de cargas parasitárias ocorreram no inverno, ao passo que os menores índices foram verificados em setembro e outubro. Com base na análise de regressão linear simples, ocorreu uma forte semelhança entre o primeiro e o terceiro anos, havendo uma tendência de influência da temperatura e da precipitação pluviomátrica sobre a carga parasitária nos animais.
\end{abstract}

Palavras-chave: Bovino, Boophilus microplus, epidemiologia, variação sazonal.

\begin{abstract}
A 3-year study of the non-parasitic and parasitic phases of the cattle tick Boophilus microplus was carried out in Botucatu, São Paulo state, Brazil. Engorged females were exposed in pastures of Brachiaria decumbens at intervals of 30 days, and left in the environment protected from sun radiation and rainfall. The preoviposition and oviposition periods were influenced by climatic changes, and there was a negative correlation with the air temperature, humidity, and rainfall. Oviposition was observed throughout the year, however between the period of May to August the eggs did not hatch. The longest and shortest larval survival periods were recorded on the progeny of ticks exposed, respectively, in August and March. The seasonal variation of $B$. microplus was studied by the counts, at intervals of 2 weeks, of engorged females on the left side of a group of nine cross-bred cattle of both sexes and approximately two years old. Peak in tick burdens were observed in winter, while the lower levels were recorded in September and October, although this variation showed, by simple linear regressive analysis, a strong similirarity just between the first and the third cycles. There was a tendency of influence of the temperature and rainfall on the burdens on the animals.
\end{abstract}

Key words: Bovine, Boophilus microplus, epidemiology, seasonal flutuation.

\footnotetext{
1 Prof. de Parasitologia Veterinária e Enfermidades Parasitárias da Universidade do Oeste Paulista (Unoeste) Hospital Veterinário da Unoeste. Rodovia Raposo Tavares Km 572 - Bairro Limoeiro - Presidente Prudente - SP - E-mail: vamilton@ vet.unoeste.br

2 Prof. Pós-Graduação da Universidade Estadual Paulista - Campus Botucatu.

* Autor para correspondência.
} 


\section{Introdução}

Os carrapatos são os mais importantes ectoparasitos das regiões tropicais e subtropicais (CASTRO; NEWSON, 1993) atingindo mais de 75\% da população mundial de bovinos (CORDOVÉS, 1997). Entre os países situados entre os paralelos $35^{0}$ Norte e $35^{0} \mathrm{Sul}$, Boophilus microplus é a espécie de carrapato de maior distribuição e importância econômica (DAVEY; OSBURN; MILLER, 1984), inclusive no Brasil, onde todo o território brasileiro é potencialmente favorável à sua sobrevivência (SOUZA et al., 1997).

A introdução de novas raças, de cruzamentos industriais de bovinos e da maior capacidade de suporte de pastagens cultivadas têm possibilitado o aumento das condições de manutenção do carrapato no Brasil (GOMES, 1998). Outro fator que tem levado ao aumento da infestação de animais no país deve-se ao uso indiscriminado de carrapaticidas, gerando uma grande pressão de seleção, que tem levado ao surgimento de cepas resistentes de B. microplus.

O conhecimento da biologia e das formas componentes do seu ecossistema, fundamentado na relação das variações ambientais com a duração da fase de vida livre e com a dinâmica populacional (NARI; SOLARI, 1991), permite definir os pontos vulneráveis do parasito, possibilitando o emprego de métodos de controle mais eficientes, como programas de controle estratégico e adequação na aplicação de drogas, assim como a integração de rotação de pastagens (HARLEY, 1966; NARI, 1995), além de fornecer dados para a simulação da dinâmica populacional por modelos matemáticos (SUTHERST; MAYWALD, 1985).

Esses argumentos fundamentaram esta pesquisa, cujo objetivo foi estudar a fase de vida livre e a flutuação sazonal do B. microplus no município de Botucatu, São Paulo, Brasil.

\section{Material e Métodos}

\section{Fase de Vida Livre}

Para o estudo da fase não parasitária do $B$. microplus na região de Botucatu, São Paulo, avaliouse os períodos de pré-postura, postura, incubação e sobrevivência larval. Mensalmente, durante o período de três anos consecutivos (setembro de 1995 a agosto de 1998), teleóginas foram retiradas de bovinos infestados naturalmente, que foram mantidos durante todo o experimento sem tratamento carrapaticida e/ ou endectocida. As fêmeas de carrapatos foram destacadas dos animais com movimentos de rotação até seu desprendimento com a finalidade de preservar a integridade de seu gnatossoma, que foi verificada ao microscópio estereoscópico, e da postura de ovos.

Após esse procedimento, 30 teleóginas foram pesadas individualmente e colocadas, cada uma, em saco de tecido de organza de aproximadamente $50 \mathrm{~cm}^{2}$. Esse recipiente, simulando o formato de um envelope, era dobrado na sua parte superior e fechado com fita adesiva, onde constavam a indicação do número da teleógina e o mês referente à colheita. Dispostos em duas caixas de madeira sobrepostas, com fundos e tampas falsos forrados com organza, os envelopes, com as fêmeas referentes ao mês de estudo, eram levados a um piquete de Brachiaria decumbens. Essa área, de $16 \mathrm{~m}^{2}$, foi cercada, para evitar a entrada de animais e permitir a ventilação das amostras, e coberta com telhado de amianto, para proteção das amostras contra ação direta da radiação solar e da precipitação atmosférica.

Foram observados os períodos de pré-postura (diariamente), postura, incubação dos ovos e sobrevivência larval (três vezes por semana) segundo os critérios adotados por Glória et al. (1993). O período de pré-postura foi considerado como aquele entre a exposição da teleógina no solo e o início da postura. $\mathrm{O}$ período de postura correspondeu à verificação do primeiro ao último ovo liberado pela fêmea, que foi considerada viva enquanto apresentou resposta aos estímulos táteis e luminosos externos. A incubação constou do tempo transcorrido entre o primeiro dia de postura e a emergência da primeira larva. 
Semanalmente, a massa de ovos era pesada em balança analítica e colocada no fundo do saco, ficando separada de cada teleógina com a fixação transversal de dois grampos de metal.

No final da postura, calculou-se o índice de conversão aparente (ICA) de cada teleógina, empregando-se a seguinte equação (BENNETT, 1974):

\section{ICA $(\%)=($ massa de ovos total $/$ peso inicial da teleógina) x 100}

Admitiram-se como ovos inférteis todos aqueles com coloração escura e aspecto de ressecamento. Para constatação de movimento das larvas, utilizaram-se dois recursos: liberação de gás carbônico e fricção dos sacos de organza para geração de calor. Caso não houvesse resposta a esses estímulos, as larvas imóveis eram consideradas mortas.

Um conjunto de teleóginas (grupo controle), pesando aproximadamente cinco gramas, foi colocado em um único saco de organza e mantido em câmara climatizada regulada à temperatura de $28^{\circ} \mathrm{C}$ e umidade relativa superior a $85 \%$. Essa operação era repetida mensalmente, no mesmo dia em que as teleóginas eram dispostas no piquete de B. decumbens, com a proposta de eliminar fatores que poderiam influenciar as variáveis estudadas, excetuando-se as intempéries ambientais.

\section{Fase de vida parasitária}

A cada 14 dias, nove bovinos mestiços de raças de origem indiana e origem européia, com predominância de sangue taurino, machos e fêmeas, idade média de dois anos, e infestados naturalmente pelo $B$. microplus, foram inspecionados no seu lado esquerdo, individualmente, para quantificação das fêmeas do carrapato maiores que 4,5 milímetros (WHARTON; UTECH, 1970).

Os animais, que durante todo o período do experimento (setembro de 1995 a agosto de 1998) permaneceram livres de tratamentos com carrapaticidas e endectocidas, foram mantidos em uma área da Fazenda Experimental da Universidade
Estadual Paulista (Unesp), Campus de Botucatu, com solo do tipo podizólico-vermelho escuro e predominância de $B$. decumbens.

\section{Dados Meteorológicos}

Índices de precipitação pluviométrica, umidade relativa do ar e temperatura, equivalentes ao período de realização do estudo, foram obtidos na Estação Meteorológica do Departamento de Ciências Ambientais da Faculdade de Ciências Agrárias da Unesp, Campus de Botucatu. A cidade está situada a $22^{0} 51^{\prime}$ sul de latitude e $48^{\circ} 26^{\prime}$ 'oeste de longitude, com altitude de 786 metros, em região constantemente úmida. Segundo o Departamento, Botucatu possui clima temperado (mesotérmico), sendo caracterizada pelos critérios de Köpen e os dados meteorológicos observados como do tipo Cfa.

\section{Análise Estatística}

Foram calculados os coeficientes de correlação linear entre as médias mensais das contagens das teleóginas, os períodos de pré-postura, postura, incubação, sobrevivência larval, o índice de conversão aparente, com cada uma das variáveis ambientais (ZAR, 1984). Para investigar a tendência do número médio de fêmeas adultas ao longo do ensaio, realizaram-se ajustes de polinômios em cada ano estudado (MEAD, 1988).

\section{Resultado e Discussão}

Os valores mínimos e máximos, as médias e os desvios-padrões das diversas etapas da fase de vida livre do B. microplus em Botucatu, São Paulo, estão registrados nas Tabelas 1 e 2 .

As fases de pré-postura e postura foram menores nos meses quentes (entre novembro e abril) e maiores nos meses frios (de maio até outubro) (Tabelas $1 \mathrm{e}$ 2, Figura 1), variando inversamente de forma significativa, com a temperatura atmosférica, a umidade relativa do ar e a precipitação pluviométrica, de acordo com a análise de correlação (Tabela 3). 
Tabela 1. Períodos mínimos (m) e máximos (M) de pré-postura (PPP), postura (PP), incubação (PI) e sobrevivência larval (SL), em dias, do Boophilus microplus na região de Botucatu, São Paulo

\begin{tabular}{lllllllll}
\hline & PPP & \multicolumn{3}{l}{ PP } & & PI & & SL \\
\cline { 2 - 8 } & $\mathrm{m}$ & $\mathrm{M}$ & $\mathrm{m}$ & $\mathrm{M}$ & $\mathrm{m}$ & $\mathrm{M}$ & $\mathrm{m}$ & $\mathrm{M}$ \\
\hline Janeiro & 3 & 7 & 16 & 45 & 29 & 96 & 27 & 113 \\
Fevereiro & 2 & 6 & 21 & 34 & 28 & 85 & 43 & 121 \\
Março & 2 & 11 & 22 & 40 & 25 & 53 & 47 & 118 \\
Abril & 2 & 8 & 19 & 44 & 29 & 49 & 37 & 93 \\
Maio & 4 & 13 & 27 & 54 & 25 & 44 & 34 & 73 \\
Junho & 3 & 13 & 23 & 51 & $\mathrm{SE}$ & $\mathrm{SE}$ & $\mathrm{SE}$ & $\mathrm{SE}$ \\
Julho & 3 & 18 & 27 & 54 & $\mathrm{SE}$ & $\mathrm{SE}$ & $\mathrm{SE}$ & $\mathrm{SE}$ \\
Agosto & 4 & 19 & 11 & 50 & 39 & 73 & 34 & 62 \\
Setembro & 4 & 12 & 24 & 54 & 32 & 128 & 29 & 57 \\
Outubro & 2 & 12 & 23 & 37 & 27 & 67 & 27 & 67 \\
Novembro & 2 & 7 & 18 & 53 & 34 & 64 & 28 & 155 \\
Dezembro & 2 & 7 & 17 & 32 & 18 & 51 & 39 & 177 \\
\hline
\end{tabular}

SE: ovos inviáveis

Tabela 2. Valores médios (c) e desvios-padrões $(S)$ dos períodos de pré-postura (PPP), postura (PP), incubação $(\mathrm{PI})$ e sobrevivência larval (SL), em dias, e do Índice de Conversão Aparente (ICA), percentual, do Boophilus microplus na região de Botucatu, São Paulo

\begin{tabular}{llllll}
\hline & $\begin{array}{l}\text { PPP (dias) } \\
(\chi \pm S)\end{array}$ & $\begin{array}{l}\text { PP (dias) } \\
(\chi \pm S)\end{array}$ & $\begin{array}{l}\text { PI (dias) } \\
(\chi \pm S)\end{array}$ & $\begin{array}{l}\text { SL (dias) } \\
(\chi \pm S)\end{array}$ & $\begin{array}{l}\text { ICA (\%) } \\
(\chi \pm S)\end{array}$ \\
\hline Janeiro & $3,8 \pm 0,7$ & $25,6 \pm 3,5$ & $40,2 \pm 4,9$ & $73,7 \pm 16,2$ & $54,9 \pm 5,3$ \\
Fevereiro & $3,3 \pm 0,8$ & $28,1 \pm 2,6$ & $41,7 \pm 8,6$ & $74,8 \pm 13,2$ & $50,2 \pm 3,7$ \\
Março & $4,5 \pm 1,3$ & $28,3 \pm 3,7$ & $37,1 \pm 4,7$ & $81,3 \pm 9,4$ & $47,4 \pm 5,2$ \\
Abril & $4,0 \pm 1,2$ & $26,1 \pm 3,7$ & $34,8 \pm 3,3$ & $72,8 \pm 9,0$ & $42,5 \pm 4,8$ \\
Maio & $7,0 \pm 1,5$ & $34,7 \pm 4,3$ & $21,0 \pm 2,0$ & $36,6 \pm 6,0$ & $33,0 \pm 5,0$ \\
Junho & $5,5 \pm 2,5$ & $32,5 \pm 4,1$ & $0,0 \pm 0,0$ & $0,0 \pm 0,0$ & $35,4 \pm 5,0$ \\
Julho & $11,0 \pm 3,2$ & $35,8 \pm 4,2$ & $0,0 \pm 0,0$ & $0,0 \pm 0,0$ & $33,5 \pm 6,0$ \\
Agosto & $7,4 \pm 2,0$ & $31,1 \pm 4,6$ & $29,1 \pm 2,1$ & $15,6 \pm 2,0$ & $41,1 \pm 3,4$ \\
Setembro & $7,2 \pm 2,0$ & $35,4 \pm 5,2$ & $34,2 \pm 6,2$ & $28,8 \pm 3,6$ & $42,7 \pm 7,5$ \\
Outubro & $5,5 \pm 1,4$ & $29,8 \pm 2,1$ & $48,1 \pm 5,4$ & $45,1 \pm 7,9$ & $49,7 \pm 5,3$ \\
Novembro & $3,9 \pm 1,0$ & $27,7 \pm 4,4$ & $47,5 \pm 4,7$ & $63,0 \pm 18,9$ & $52,4 \pm 6,1$ \\
Dezembro & $3,6 \pm 0,7$ & $23,3 \pm 2,1$ & $36,4 \pm 4,0$ & $75,2 \pm 15,7$ & $48,8 \pm 7,5$ \\
\hline
\end{tabular}

Tabela 3. Coeficientes de variação dos períodos de pré-postura (PPP), postura (PP), incubação (PI) e sobrevivência larval (SL), e o índice de conversão aparente (ICA) do Boophilus microplus, na região de Botucatu, São Paulo, com a temperatura $\left({ }^{0} \mathrm{C}\right)$, umidade relativa do $\operatorname{ar}(\%)$ e precipitação pluviométrica $\left(\mathrm{mm}^{3}\right)$

\begin{tabular}{llllll}
\hline & \multicolumn{2}{l}{ Variáveis Estudadas } & & \\
Variáveis Ambientais & PPP & PP & PI & SL & ICA \\
\hline Temperatura & $-0,5441^{*}$ & $-0,5763^{*}$ & 0,3710 & $0,6900^{*}$ & $0,6335^{*}$ \\
Umidade Relativa do Ar & $-0,5807^{*}$ & $-0,5663^{*}$ & 0,0349 & 0,4358 & $0,4936^{*}$ \\
Precipitação Pluviométrica & $-0,4313^{*}$ & $-0,5306^{*}$ & 0,2308 & $0,5009^{*}$ & $0,6679^{*}$ \\
\hline
\end{tabular}

* coeficiente de correlação significativo $(\mathrm{p}<0,05)$ 


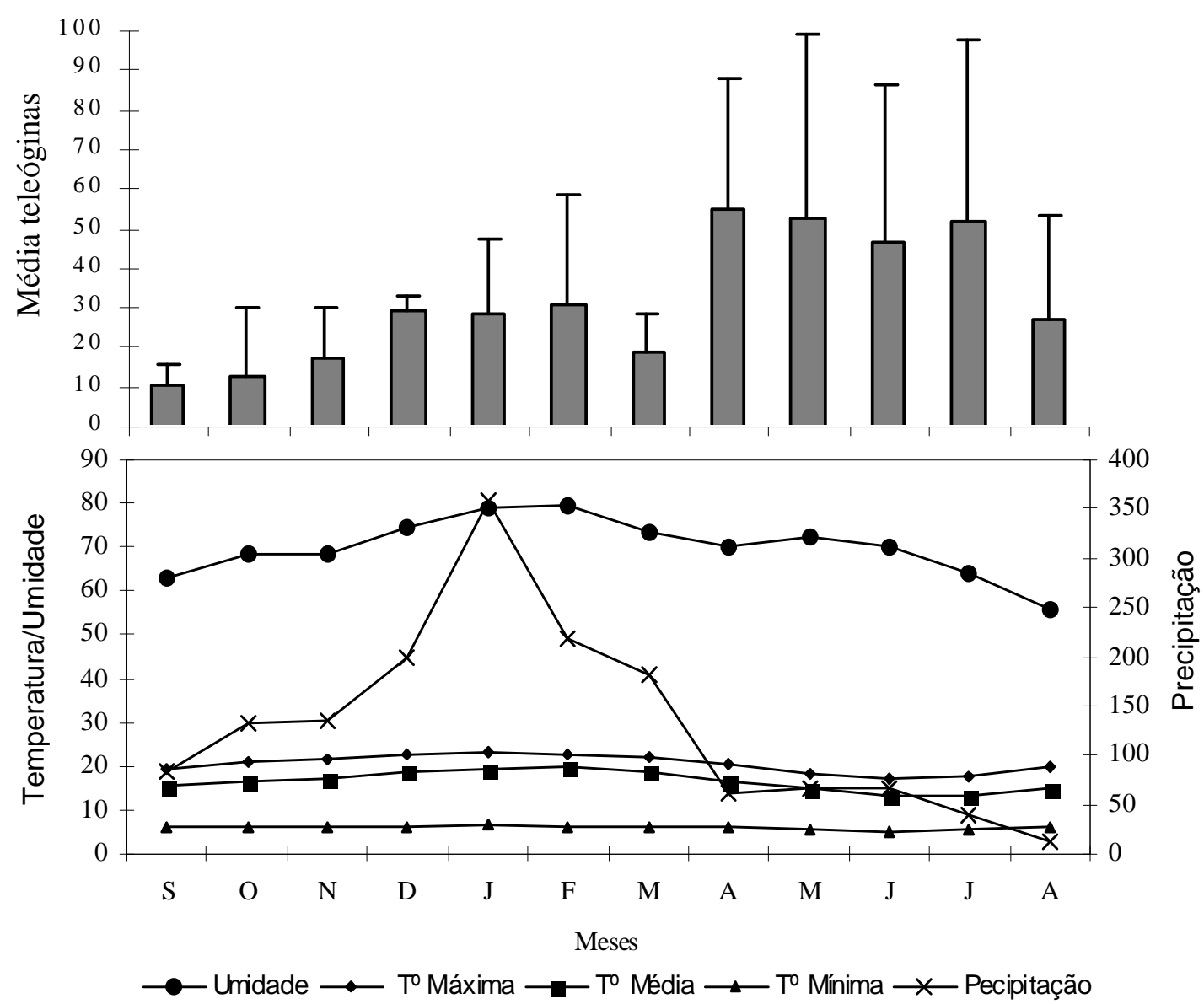

Figura 1. Contagem média de teleóginas em bovinos e médias das temperaturas mínima, média e máxima $\left({ }^{\circ} \mathrm{C}\right)$, da umidade relativa do ar (\%) e da precipitação pluviométrica $\left(\mathrm{mm}^{3}\right)$, em três anos consecutivos, no município de Botucatu, São Paulo, Brasil

Esses achados corroboram Oliveira et al. (1974), que verificaram a existência de correlação negativa entre temperatura e precipitação pluviométrica com a pré-postura, postura e pré-eclosão, e Gomes (1998), que constatou a influência da temperatura e umidade sobre os dois primeiros períodos. Os resultados obtidos por Gonzales et al. (1975), Souza et al. (1988a), Magalhães e Lima (1992), Almeida et al. (1994a), em campo, por Rocha et al. (1985), Davey, Cooksey e Despins (1991) e Glória et al. (1993), em laboratório, determinaram a temperatura como único fator ambiental que interferiu nestas variáveis. Contudo, esses autores trabalharam em condições de umidade favoráveis às necessidades biológicas do $B$. microplus, não observando as interferências da precipitação pluviométrica.
Embora houvesse ocorrido postura em todos os meses do ano, os ovos provenientes de teleóginas expostas em maio a agosto apresentavam características de infertilidade (Tabela 1). Este fato foi observado por Souza et al. (1988a) nos meses de abril a agosto, e por Brum, Gonzales e Petruzzi (1985) e Laranja et al. (1986) nos meses de maio e julho. Nos estudos de Paloschi e Beck (1989), Almeida et al. (1994a) e Saueressig (1994), as condições climáticas foram favoráveis à eclosão de larvas durante todo o ano.

Davey, Cooksey e Despins (1991), em condições laboratoriais, empregando várias combinações de temperatura e umidade, concluíram que essa última variável, quando abaixo de 63\%, foi responsável pela 
infertilidade dos ovos independentemente da temperatura empregada. Em condições controladas, Rocha et al. (1985) determinaram que a postura e a embriogênese foram favorecidas pela umidificação do ar. Hitchcock (1955) e Moraes et al. (1987) observaram redução de ovos férteis em baixas temperaturas, e Bennett (1974), em situações de déficites de umidade.

Verificou-se nesse estudo, que entre junho e agosto as baixas temperaturas e umidades, que alcançaram índices abaixo de $60 \%$, propiciaram a dessecação dos ovos, fato também reportado por Gonzales et al. (1975) e por Brum, Gonzales e Petruzzi (1985).

Os maiores e os menores percentuais de ICA, foram obtidos, respectivamente, nos meses quentes e frios (Tabela 2), e estão de acordo com os achados de Souza et al. (1988a). Houve correlação positiva, de forma significativa, entre a conversão aparente de ovos e a temperatura, a umidade e a precipitação atmosférica (Tabela 3). Observou-se também que o percentual de ovos férteis foi diretamente proporcional ao ICA. O mesmo foi descrito por Veríssimo (1987), ao verificar que quando a massa de ovos é grande, existe uma passagem de umidade dos ovos da superfície para aqueles localizados no interior da massa, aumentando as chances de eclosão das larvas, mesmo que as condições ambientais sejam adversas ao desenvolvimento embrionário.

O tempo máximo de incubação registrou-se quando o mês de exposição de fêmeas ocorreu em setembro e os menores períodos foram obtidos em março e abril. Nessa variável, a temperatura foi o componente climático de maior influência, apresentando correlação positiva com os meses em que ocorreu a incubação.

Quanto a sobrevivência das larvas, observou-se que as progênies provenientes de fêmeas colocadas em canteiros nos meses quentes, compreendidos entre dezembro e março, apresentaram maior longevidade. Este fato ocorreu como conseqüência das baixas temperaturas dos meses de inverno, de maio a agosto, a que foram submetidas, confirmando os achados de Gonzales et al. (1975), Souza et al. (1988a) e Souza e Serra Freire (1991) e Candido et al. (1983) consideraram que os altos níveis de umidade é responsável pela manutenção de larvas nas pastagens, fato não observado nesse estudo.

A menor longevidade foi das larvas nascidas no início dos meses quentes, de dezembro a março, e originadas de fêmeas expostas em agosto, setembro e outubro, como conseqüência da influência negativa da alta temperatura. Esse fato foi descrito também por Candido et al. (1983), Magalhães e Lima (1992) e Braga (1994). Segundo Gonzales et al. (1975), o calor acelera o metabolismo das larvas, levando-as à morte por esgotamento energético. Essas afirmações, contudo, vão de encontro aos resultados obtidos por Hitchcock (1955) e Davey e Cooksey (1989), que atribuíram a suscetibilidade de larvas às reduções térmicas em condições controladas de laboratório.

Durante o transcorrer do ensaio, as fêmeas mantidas em estufa incubadora produziram postura fértil, com incubação média de 29 dias, permitindo afirmar que as intempéries ambientais influenciaram a fase de vida livre.

Quanto à fase parasitária, verificou-se, pela análise de regressão linear, que ocorreu uma variação nos três ciclos de estudo, sendo as contagens no primeiro e no terceiro anos bastate semelhantes. Sutherst et al. (1983) também notaram diferença na distribuição populacional de B. microplus em um mesmo ambiente com estudos em diferentes períodos.

A infestação dos animais foi maior nos meses frios e de baixa precipitação, chegando a valores mínimos em setembro (Figura 1). Contudo, Arteche e Laranja (1979) e Saueressig e Honer (1993) apontaram a temperatura como único fator que exerceu influência, de forma positiva, sobre a contagem de teleóginas nos animais.

Os resultados desse estudo são semelhantes aos de Alves-Branco, Pinheiro e Sapper (1989) e Martins et al. (1995), RS, que observaram as maiores infestações em bovinos nas estações frias, mas são 
contrários aos achados de Guaragna et al. (1988), na Índia; Souza et al. (1988b), SC; Fonseca, Pereira e Mafra (1993), MG; Silva (1993), Pr; Saueressig e Honer (1993), DF; Almeida et al. (1994b), Ba; e Gomes et al. (1989), MS, que registraram menor

Sutherst et al. (1973), Sutherst et al. (1983) e Vianna, Faccini e Pereira (1995) consideraram que o aumento de carrapatos nos bovinos nos meses frios pode ser decorrente de uma diminuição da resistência dos animais em virtude da baixa oferta de alimentos, infestação no inverno, levando-os, como consequiência, a uma maior suscetibilidade à fixação do carrapato.

Observou-se no presente trabalho que na região de Botucatu, os meses quentes foram desfavoráveis à sobrevivência de larvas, favorecendo o emprego de rotação de pastagens como medida alternativa para o controle do B. microplus no verão. Já os meses frios proporcionaram condições de sobrevivência de larvas e altas infestações pela maior suscetibilidade dos animais pela baixa oferta de alimentos resultante das baixas temperaturas, umidades e precipitação pluviométrica.

O controle estratégico desse ectoparasito em Botucatu poderia ser formulado com banhos carrapaticidas aplicados a partir do meado de fevereiro, em intervalos quinzenais, até o fim de abril, a fim de se evitar a alta infestação de animais no inverno, uma vez que as larvas oriundas principalmente das teleóginas caídas ao solo no outono são responsáveis pela contaminação da pastagem no período frio.

\section{Referências}

ALMEIDA, M. A. O.; ARAÚJO, F. R.; CARVALHO, E. L. L.; SANTARÉM, V. A.; LEAL, C. R. B.; JULIÃO, F. S. Fase de vida livre do Boophilus microplus no município de Salvador, Bahia. In: CONGRESSO BRASILEIRO DE MEDICINA VETERINÁRIA, 23., 1994, Olinda. Anais... Olinda: Sociedade Brasileira de Medicina Veterinária, 1994a. p. 246.
ALMEIDA, M. A. O.; TORRES, P.E. L. M. V.; CARVALHO, E. L. L.; ARAÚJO, F. R., SANTARÉM, V. A. Flutuação estacional do Boophilus microplus no Litoral Norte Baiano. In: CONGRESSO BRASILEIRO DE MEDICINA VETERINÁRIA, 23., 1994, Olinda. Anais... Olinda: Sociedade Brasileira de Medicina Veterinária, 1994b. p. 247.

ALVES-BRANCO, F. P. J.; PINHEIRO, A. C.; SAPPER, M. F. M. Controle do Boophilus microplus com esquemas de banhos estratégicos em bovinos Hereford. Bagé: EMBRAPA, 1989. (Circular Técnica, 4).

ARTECHE, C. C. P.; LARANJA, R. J. Epidemiologia do Boophilus microplus (Can. 1888): incidência sazonal no Sudeste do Rio Grande do Sul. Boleteim do Instituto de Pesquisas Veterinárias "Disiderio Finamor", Porto Alegre, v. 6, p. 29-43, 1979.

BENNETT, G. F. Oviposition of Boophilus microplus (Canestrini) (Acarida: Ixodidae). II. Influence of temperature, humidity and light. Acarologia, Paris, v.16, p.250-7, 1974.

BRAGA, R. M. Ecologia do carrapato dos bovinos em Roraima. In: CONGRESSO BRASILEIRO DE MEDICINA VETERINÁRIA, 23., 1994, Olinda. Anais... Olinda: Sociedade Brasileira de Medicina Veterinária, 1994. p. 334.

BRUM, J. G. W.; GONZALES, J. C.; PETRUZZI, M. A. Postura e eclosão de Boophilus microplus (Can. 1887) em diferentes localizações geográficas do Rio Grande do Sul, Brasil. Arquivos Brasileiros de Medicina Veterinária e Zootecnia, Belo Horizonte, v.37, p.581-7, 1985.

CANDIDO, I. C.; HATAYDE, M. R.; VASCONCELOS, O. T.; ROCHA,U. F.; WOELZ, C. R. Ecologia de carrapatos. IV. Sobrevivência de larvas de Boophilus microplus em laboratório e no campo. In: CONGRESSO DA FEDERACION LATINOAMERICANA DE PARASITOLOGIA, 6., 1983, São Paulo. Resumos... São Paulo: Sociedade Brasileira de Parasitologia, 1983. p. 273.

CASTRO, J. J.; NEWSON, R. M. Host resistance in cattle tick control. Parasitology Today, Limerick, v.9, p.13-7, 1993.

CORDOVÉS, C. O. Carrapato: controle ou erradicação. 2.ed. Guaíba: Agropecuária, 1997.

DAVEY, R. B.; COOKSEY, L. M. Effects of prolonged exposure at low temperature on Boophilus microplus (Acari: Ixodidae). Journal Medical of Entomology, Lanham, v.26, p.407-410, 1989.

DAVEY, R. B.; COOKSEY, L. M.; DESPINS, J. L. Survival of larvae of Boophilus annulatus, Boophilus microplus, and Boophilus hibrids (Acari: Ixodidae) in different temperature and humidity regimes in the laboratory. Veterinary Parasitology, Amsterdam, v.40, p.305-13, 1991. 
DAVEY, R. B.; OSBURN, R.L.; MILLER, J. A. Ovipositional and morphological comparisions of Boophilus microplus (Acari: Ixodidae) collected from different geographic areas. Annals of the Entomological Society of America, Lanham, v.77, p.1-5, 1984.

FONSECA, A. H.; PEREIRA, M. J. S.; MAFRA, C. L. Dinâmica populacional do carrapato Boophilus microplus em São Miguel do Anta - MG, Brasil. Revista Brasileira de Parasitologia Veterinária, São Paulo, v.6, p.121, 1993.

GLÓRIA, M. A.; FACCINI, J. L. H.; DAEMON, E.; GRISI, L. Influência de diferentes temperaturas sobre a biologia da fase não parasitária de Boophilus microplus (Can. 1887) (Acari: Ixodidae). Revista Brasileira de Parasitologia Veterinária, São Paulo, v.2, p.85-91, 1993.

GOMES, A. O carrapato-do-boi Boophilus microplus: ciclo, biologia, epidemiologia, patogenia e controle. In: KESSLER, R. H.; SCHENK, M. A. M. Carrapato, Tristeza Parasitária e Tripanossomose dos bovinos. Campo Grande: EMBRAPA, 1998. p. 9-44.

GOMES, A.; HONER, M. R.; SCHENK, M. A.; CURVO, J. B. E. Populations of the cattle tick (Boophilus microplus) on purebred Nellore, Ibagé and Nellore X European crossbreds in the Brazilian Savanna. Tropical Animal Health and Production, Dordrecht, v.21, p.20-24, 1989.

GONZALES, J. C.; SILVA, N. R.; FRANCO, N.; PEREIRA, I. H. O. A vida livre do Boophilus microplus (Can. 1887). Arquivos da Faculdade de Veterinária da UFRGS, Porto Alegre, v.3, p.21-28, 1975.

GUARAGNA, G. P.; CARVALHO, J. B. P.; FIGUEIREDO, A. L.; GAMBINI, L. B.; BARBOSA, M. I. A. Efeito de fatores genéticos e ambientais na infestação natural de carrapatos (Boophilus microplus, Canestrini) em bovinos leiteiros. Boletim de Industria Animal, Nova Odessa, v.45, p.19-32, 1988.

HARLEY, K. L. S. Studies on the survival of the nonparasitic stages of the cattle tick Boophilus microplus in three climatically dissimilar districts of North Queensland. Australian Journal of Agricultural Research, Colligwood, v.7, p. 387-410, 1966.

HITCHCOCK, L. F. Studies on the non-parasitic stages on the cattle tick, Boophilus microplus (Canestrini) (Acarina: Ixodidae). Australian Journal of Zoology, Colligwood, v. 3, p. 295-311, 1955.

LARANJA, R. J.; CERESÉR, V. H.; MARTINS, J. R.; CASTAGNA, M.; FERREIRA, F.; EVANS, D. E. Potencial de reprodução do Boophilus microplus na Região de Campos de Cima da Serra, Vacaria, RS. Boletim do Instituto de Pesquisas Veterinárias “Desiderio Finamor", Porto Alegre, v.1, p.9-13, 1986.
MAGALHÃES, F. E. P.; LIMA, J. D. Desenvolvimento e sobrevivência do carrapato em pastagem de Brachiaria decumbens no município de Pedro Leopoldo, MG. Pesquisa Agropopecuária Brasileira, Brasília, v.27, p.1525, 1992.

MARTINS, J. R.; CERESÉR, V. H.; CORREA, L. B.; ARTECHE, C. C. P. O controle correto do carrapato. Porto Alegre: Fepagro, 1995. (Circular Técnica, 5).

MEAD, R. The design of experiments: statistical principles for pratical application. Cambridge: Cambridge University, 1988.

MORAES, F. R.; SILVA, M. S.; MORAES, J. R. E.; FREITAS, J. C. M.; ROCHA, U. F. Ecologia de carrapatos. XXInfluência do abaixamento brusco da temperatura, e de sua manutenção por cinco dias, sobre desenvolvimento embrionário e eclosão larval de Boophilus microplus (Canestrini). Ars Veterinária, Jaboticabal, v. 3, p. 89-95, 1987.

NARI, A. Strategies for the control of one-host ticks and relationship with tick-borne diseases in South America. Veterinary Parasitology, Amsterdam, v.57, p.153-65, 1995.

NARI, A.; SOLARI, M. A. Epidemiología y control del Boophilus microplus en Uruguay. Su relación com Babesia spp. Revista Cubana de Ciencias Veterinartia, La Habana, v.22, p.149-60, 1991.

OliveirA, G. P.; COSTA, R. P.; MELlO, R. P.; MENEGUELLI, C. A. Estudo ecológico da fase não parasitária do Boophilus microplus (Canestrini, 1887) (Acarina, Ixodidae) no Estado do Rio de Janeiro. Arquivos Universidade Federal Rural do Rio de Janeiro, Rio de Janeiro, v.4, p.1-10, 1974.

PALOSCHI, C. G.; BECK, A. A. H. Fase de vida livre do Boophilus microplus (Canestrini, 1887) no Vale do Itajaí, SC. In: SEMINÁRIO BRASILEIRODEPARASITOLOGIA VETERINÁRIA, 6., 1989, Bagé. Anais... Bagé: Colégio Brasileiro de Parasitologia Veterinária, 1989. p.72.

ROCHA, U. F.; COSTA, A. J.; FERREIRA, F. A.; MACIEL, R. A. Ecologia de carrapatos. X. Câmara úmida improvisada para estudos de influências de umidade atmosférica e da suscetibilidade individual de bovinos sobre oviposição e embriogênese de Boophilus microplus. Veterinaria $e$ Zootecnia, São Paulo, v.1, p.73-87, 1985.

SAUERESSIG, T. M. Estudo da fase não parasitária do carrapato de bovino em pastagens cultivadas e nativa do Distrito Federal. Planaltina: EMBRAPA-CPAC, 1994. (Boletim de Pesquisa, 37).

SAUERESSIG, T. M.; HONER, M. R. Dinâmica populacional do carrapato Boophilus microplus nos cerrados do Distrito Federal: análises e simulaçãoes. In: SEMINÁRIO BRASILEIRO DE PARASITOLOGIA VETERINÁRIA, 8., 1993, Londrina. Anais... Londrina: Colégio Brasileiro de Parasitologia Veterinária, 1993. p. A3. 
SILVA, N. L.; PEROTTO, D.; CUBAS, A. C.; LESSKIU, C.; MOLETTA, J. L.; MOTTA, J. B. O. Modelo populacional do Boophilus microplus (Canestrini, 1887) em bovinos de corte, na região Sul do Estado do Paraná. In: SEMINÁRIO BRASILEIRO DE PARASITOLOGIA VETERINÁRIA, 7., 1993, Londrina. Anais... Londrina: Sociedade Rural do Paraná, 1993. p. A1.

SOUZA, A. P.; GONZALES, J. C.; RAMOS, C. I.; PALOSCHI, C. G.; MORAES, A. N. Fase de vida livre do Boophilus microplus no Planalto Catarinense. Pesquisa Agropecuária Brasileira, Brasília, v. 23, p. 427-34, 1988a.

SOUZA, A. P.; GONZALES, J. C.; RAMOS, C. I.; PALOSCHI, C. G.; MORAES, A. N. Variação sazonal de Boophilus microplus no Planalto Catarinense. Pesquisa Agropecuária Brasileira, Brasília, v. 23, p. 627-30, 1988 b.

SOUZA, A. P.; SERRA-FREIRE, N. M. Variação sazonal da fase não-parasitária de Amblyomma cajennense e Boophilus microplus no município de Paracambi, RJ. Revista Brasileira Parasitologia de Veterinária, São Paulo, v. 1, p. 44, 1991.

SOUZA, E. J.; PERALVA, S. L. F. S.; REIS, R. C. S.; BITTENCOURT, V. R. E. P. Avaliação da eficácia do fungo Metharizium anisopliae (METSCHNIKOFF, 1879) Sorokin, 1883 em teste de campo com bovinos infestados com carrapato Boophilus microplus (Canestrini, 1887) (Acari: Ixodidae). Revista Brasileira de Parasitologia Veterinária, São Paulo, v. 6, p. 109, 1997.
SUTHERST, R. W.; UTECH, K. B. W.; DALLWITZ, M. J.; KERR, J.D. Intraspecific competition of Boophilus microplus (Canestrini) on cattle. Journal Applied of Ecology, Oxford,v.10, p.855-62, 1973.

SUTHERST, R. W.; KERR, J. D.; MAYWALD, G. F.; STEGEMAN, D. A. The effect of season and nutrition on the resistance of cattle to the tick Boophilus microplus. Australian Journal of Agricultural Research, Colligwood, v.34, p.329-39, 1983.

SUTHERST, R. W.; MAYWALD, G. F., A computerised system for matching climates in ecology. Agriculture, Ecosystems \& Envinroment, Amsterdam, v. 13, p. 281-9, 1985.

VERÍSSIMO, C. J. Relatório de estágio sobre o carrapato Boophilus microplus. Zootecnia, São Paulo, v. 25, p.295$332,1987$.

VIANNA, L. F. C. G.; FACCINI, J. L. H.; PEREIRA, M. B. Susceptibilidade de bovinos mestiços ao carrapato Boophilus microplus (Canestrini, 1887) na microrregião Fluminense do Grande Rio, Estado do Rio de Janeiro. Revista Brasileira de Ciências Veterinária, Niterói, v.2, p. 43-6, 1995.

WHARTON, R. H.; UTECH, K. B. W. The relation between engorgement and dropping of Boophilus microplus (Canestrini) (Ixodidae) to the assessment of tick numbers on cattle. Journal of the Australian Entomological Society, Canberra, v. 9, p. 171-82, 1970.

ZAR, J. H. Biostatistical Analysis. $2^{\text {th }}$ ed. Englwood Cliffs: Prentice-Hall, 1984. 
